\title{
Numerical Comparisons of Expected Log-Utility Maximization Problem with a Factor Model under Several Situations
}

\author{
Y. Asakura \\ Graduate School of Science and Engineering, Hosei University \\ 3-7-2, Kajino-cho, Koganei-shi, Tokyo, 184-8584, Japan \\ E-mail: yuya.asakura.2i@stu.hosei.ac.jp \\ Kazuhiro Yasuda \\ Faculty of Science and Engineering, Hosei University \\ 3-7-2, Kajino-cho, Koganei-shi, Tokyo, 184-8584, Japan \\ E-mail: k_yasuda@hosei.ac.jp
}

\begin{abstract}
In this paper, we consider effects of information, estimations and constraints on a portfolio optimization problem in mathematical finance. In particular, a portfolio optimization problem of investors who want to maximize their expected utility of their terminal wealth is considered. As our risky security model we adopt a factor model in which the growth rate depends on an exogenous factor. We assume several strategies whose differences come from information of markets, estimations of the factor and constraints of strategies, and we numerically investigate their effects on the expected utility. The logarithmic utility function as the utility function showing risk aversion investors is used in their paper.
\end{abstract}

\section{Introduction}

In this paper, we consider effects of information, estimations and constraints on a portfolio optimization problem in mathematical finance. Portfolio optimization problems of investors who want to maximize their expected utility of their terminal wealth have been widely studied under various situations, e.g. Karatzas, Lehoczky, Shreve and Xu [3], Cvitanić and Karatzas [2], Lakner [4], [5], Sass [7], Putschögl and Sass [6], and so on. However most of these studies only drive their optimal solutions under each situation, they do not enough compare among results. In fact, there exist few papers which discuss how much differences appear in expected utilities under different situations, e.g. Yang, Ewald and Wang [8] as rare papers. In this article, we would like to numerically show how much differences appear in certainty equivalents of terminal expected utilities from investor's different situations. Through these results, we can find values of information, costs of estimations, and losses from restrictions and so on. Preliminary results are given in Asakura and Yasuda [1].
In this paper, as our risky security model we adopt a factor model in which the growth rate depends on an exogenous factor, which here follows the Ornstein-Uhlenbeck process. Under the market model, we assume various strategies whose differences come from information of markets, estimations of the factor and constraints of strategies, and we numerically show their effects on the expected utility, precisely speaking we compare them through their certainty equivalents. We use the logarithmic utility function as the utility function showing risk aversion investors.

In this article we also give histograms with respect to terminal wealth and fundamental statistics of log-return at the maturity in order to understand features of each strategy. Finally we give scenario based results, that is, ranking of each simulation scenario of terminal wealth in a group of strategies, and standing of each simulation scenario of terminal wealth between each two strategies. They show scenario based interpretations of each strategy.

This paper is organized as follows: in Section 2, we will give settings of our markets and define strategies under several situations, and in Section 3, we will give some numerical results, compare difference among strategies and give some fundamental statistics of terminal wealth and so on.

\section{Settings}

\subsection{Settings of markets}

Let $\left(\Omega, \mathcal{F},\left\{\mathcal{F}_{t}\right\}_{0 \leq t \leq T}, P\right)$ be a filtered complete probability space, where we assume that the filtration $\mathcal{F}_{t}$ satisfies the usual condition. We assume that in our financial market there are a risk-free asset and a risky security whose growth rate follows the Ornstein-Uhlenbeck process. That is, the price process of the risky security $S=\left\{S_{t}\right\}_{0 \leq t \leq T}$ and the expected return process $\mu=\left\{\mu_{t}\right\}_{0 \leq t \leq T}$ are determined by the 
following stochastic differential equations:

$$
\begin{aligned}
d S_{t} & =\mu_{t} S_{t} d t+\sigma S_{t} d w_{t}^{(1)}, \\
d \mu_{t} & =\alpha\left(v-\mu_{t}\right) d t+\beta d w_{t}^{(2)},
\end{aligned}
$$

where $S_{0}(>0)$ and $\mu_{0}$ are initial values of the risky security and the expected return respectively, the mean-reverting level $v$ is a constant, and $\sigma(>0)$ is the volatility of the security price process, $\alpha(>0)$ is the mean-reverting speed and $\beta(>0)$ is the volatility of the factor. The processes $w^{(1)}=$ $\left\{w_{t}^{(1)}\right\}_{0 \leq t \leq T}$ and $w^{(2)}=\left\{w_{t}^{(2)}\right\}_{0 \leq t \leq T}$ are independent two Brownian motions on the probability space $\left(\Omega, \mathcal{F},\left\{\mathcal{F}_{t}\right\}_{0 \leq t \leq T}, P\right)$. Note that in our model, the risky security price $S_{t}$ of course depends on the factor $\mu_{t}$, the factor $\mu_{t}$ is, however, independent of the risky security price $S_{t}$. Finally let $B=\left\{B_{t}\right\}_{0 \leq t \leq T}$ be the risk-free asset and we assume that $B_{t}$ follows the ordinary differential equation:

$$
d B_{t}=r B_{t} d t
$$

where $r(>0)$ is the interest rate of the risk-free asset.

Let a trading strategy $\pi=\left\{\pi_{t}\right\}_{0 \leq t \leq T}$ be the amount of money invested in the risky security $S$ and $X^{\pi}=\left\{X_{t}^{\pi}\right\}_{0 \leq t \leq T}$ be the wealth process of the investor with the trading strategy $\pi$. We shall assume that the trading strategy $\pi_{t}$ satisfies the self-financing, that is,

$$
d X_{t}^{\pi}=\pi_{t} \frac{d S_{t}}{S_{t}}+\left(X_{t}^{\pi}-\pi_{t}\right) \frac{d B_{t}}{B_{t}},
$$

and admissible i.e. $X_{t}^{\pi} \geq 0$ a.s. for all $t \in[0, T]$. Let $X_{0}(>0)$ be an initial wealth. We denote $U(x)$ as a utility function of risk aversion's investors. We assume that $U\left(\in C^{1}\right)$ is a strictly increasing and strictly concave function with $\lim _{x \rightarrow \infty} U^{\prime}(x)=0$. In this paper, we consider the logarithmic function $U(x)=\log x$ as the utility function. Finally the expected log-utility maximization problem is defined as

$$
\max _{\pi} E\left[\log X_{T}^{\pi}\right]
$$

under various conditions with respect to the strategy $\pi$, which will be explained in the following subsection. Then certainty equivalent $x_{*}$ with respect to the maximization problem is defined as

$$
\log x_{*}=\max _{\pi} E\left[\log X_{T}^{\pi}\right]
$$

Through $x_{*}$, we can find the values of information, costs of estimations, losses from restrictions and so on.

\subsection{Settings of strategies}

In this subsection, we give the following six trading strategies which are treated and compared in this paper. We assume that the wealth process with each strategy has enough integrabilities.

(i). Strategy 1: In Strategy 1, we assume that the investor has the filtration $\mathcal{F}_{t}^{S, \mu}=\sigma\left(S_{s}, \mu_{s} ; 0 \leq s \leq t\right)$, that is, the investor has both information of the security price $S$ and the factor value $\mu$. We call this situation full information. From Example 10.1 of Karatzas, Lehoczky, Shreve and Xu [3], the optimal strategy under full information with respect to the factor market model (1) is $\left\{\mathcal{F}_{t}^{S, \mu}\right\}$-adapted and is given as

$$
\pi_{t}^{F}=\frac{\mu_{t}-r}{\sigma^{2}} \hat{X}_{t}^{F}
$$

where $\hat{X}^{F}$ is the wealth process with strategy $\pi_{t}^{F}$ (Strategy 1).

(ii). Strategy 2: In Strategy 2, we assume that the investor has the filtration $\mathcal{F}_{t}^{S}=\sigma\left(S_{s} ; 0 \leq s \leq t\right)$, that is, the investor has only information of the security price $S$ and does not have information of the factor $\mu$. We call this situation partial information. From Example 4.4 of Lakner [4], the optimal strategy under partial information with respect to the factor market model (1) is $\left\{\mathcal{F}_{t}^{S}\right\}$-adapted and is given as

$$
\pi_{t}^{P}=\frac{m_{t}-r}{\sigma^{2}} \hat{X}_{t}^{P},
$$

where $m_{t}$ is defined as $m_{t}=E\left[\mu_{t} \mid \mathcal{F}_{t}^{S}\right]$ and $\hat{X}^{P}$ denotes the wealth process with strategy $\pi_{t}^{P}$ (Strategy 2). In order to obtain an explicit expression of $m_{t}$, we can use linear Gaussian filtering theory. From Eqs.(4.6) and (4.7) in Lakner [4], $m_{t}$ is known as the unique solution of

$$
d m_{t}=-\left(\alpha+\frac{\gamma(t)}{\sigma^{2}}\right) m_{t} d t+\frac{\gamma(t)}{\sigma^{2}} d R_{t}+\alpha v d t,
$$

where $\gamma(t)$ is the unique solution of the Riccati equation:

$$
d \gamma(t)=\left(-\frac{\gamma(t)^{2}}{\sigma^{2}}-2 \alpha \gamma(t)+\beta^{2}\right) d t
$$

and $R_{t}$ is defined as $d R_{t}=\mu_{t} d t+\sigma d w_{t}^{(1)}$. Note that $\gamma(t)$ is originally defined as $\gamma(t)=E\left[\left(\mu_{t}-m_{t}\right)^{2} \mid \mathcal{F}_{t}^{S}\right]$ in Eq.(3.2) of Lakner [4] and the explicit representations of $m_{t}$ and $\gamma(t)$ are also given in Eq.(4.9) and Remark 4.2 of Lakner [4] respectively.

(iii). Strategy 3: In Strategy 3, we assume that the investor does not observe factor values, namely under partial information, and suppose that the growth rate is a fixed constant rate, that is, the investor does not estimate the factor like Strategy 2. In this sense, this investor does not use any information, and believes and invests using the investor's intuition. In Strategy 3 , as the constant, we use $\mu_{0}$ which is the initial value of the factor process. Then, Strategy 3 is defined as

$$
\pi_{t}^{\mu_{0}}=\frac{\mu_{0}-r}{\sigma^{2}} X_{t}^{\mu_{0}}
$$

where $X^{\mu_{0}}$ is the wealth process with $\pi_{t}^{\mu_{0}}$ (Strategy 3). This strategy is not an optimal strategy under the factor model (1). 
(iv). Strategy 4: As Strategy 3, the investor supposes that the growth rate is a fixed constant rate. In Strategy 4, as the investor's intuition, we use $v$ which is the reverting level of the factor process. Then, our strategy is

$$
\pi_{t}^{v}=\frac{v-r}{\sigma^{2}} X_{t}^{v}
$$

as Strategy 4 , where $X^{v}$ is the wealth processes with Strategy 4. This strategy is not an optimal strategy under the factor model (1). However using the mean reverting level in the strategy, this investor can be regarded as having a good intuition.

(v). Strategy 5: We consider the case with upper and lower restrictions with respect to the amount of the risky security under full information. From 14.8 EXAMPLE of Cvitanić and Karatzas [2], the optimal strategy under full information is given as

$$
\begin{aligned}
\pi_{t}^{F C}= & u \hat{X}_{t}^{F C} \mathbf{I}\left\{\frac{\mu_{t}-r}{\sigma^{2}}>u\right\} \\
& +\frac{\mu_{t}-r}{\sigma^{2}} \hat{X}_{t}^{F C} \mathbf{I}\left\{l \leq \frac{\mu_{t}-r}{\sigma^{2}} \leq u\right\} \\
& +l \hat{X}_{t}^{F C} \mathbf{I}\left\{\frac{\mu_{t}-r}{\sigma^{2}}<l\right\},
\end{aligned}
$$

where $\mathbf{I}$ denotes the indicator function, $u$ and $l$ denotes risky security proportions of upper and lower bound respectively and $\hat{X}^{F C}$ is the wealth process with Strategy 5 .

(vi). Strategy 6: As Strategy 5, we consider the case with upper and lower restrictions with respect to the amount of the risky security under partial information. From Lemma 3.3 in Sass [7] and Example 4 in Putschögl and Sass [6], the optimal strategy under partial information is given as

$$
\begin{aligned}
\pi_{t}^{P C}= & u \hat{X}_{t}^{P C} \mathbf{I}\left\{\frac{m_{t}-r}{\sigma^{2}}>u\right\} \\
& +\frac{m_{t}-r}{\sigma^{2}} \hat{X}_{t}^{P C} \mathbf{I}\left\{l \leq \frac{m_{t}-r}{\sigma^{2}} \leq u\right\} \\
& +l \hat{X}_{t}^{P C} \mathbf{I}\left\{\frac{m_{t}-r}{\sigma^{2}}<l\right\},
\end{aligned}
$$

where $u$ and $l$ denotes risky security proportions of upper and lower bound respectively and $\hat{X}^{P C}$ is the wealth process with Strategy 6.

\section{Numerical results}

\subsection{Parameter settings and simulation method}

The expected utility under each strategy is simulated using the following parameter values:

$$
\begin{aligned}
& S_{0}=100, \mu_{0}=0.02, X_{0}=500, T=1, r=0.03, \\
& \sigma=0.3, \alpha=2, v=0.3, \beta=0.5, u=6, l=-1,
\end{aligned}
$$

In order to simulate stochastic differential equations, we use the Euler-Maruyama approximation with 300 time steps, and also for an approximation of the expectation, we use $10^{5}$ times Monte-Carlo simulation.

\subsection{Effects of each parameter on expected utilities}

In this section, we compare certainty equivalent at the terminal time among Strategy 1-6. Interpretations of differences of certainty equivalents among strategies are considered as follows:

(i) Differences of certainty equivalents between Strategy 1 and 2 can be regarded as information value since Strategy 1 is the best (optimal) strategy of investors who have full information, Strategy 2 is the best (optimal) strategy of investors who have partial information, and the difference between two strategies is only the difference of information which investors have.

(ii) Differences of certainty equivalents between Strategy 2 and 3-4 can be regarded as costs of estimation since investors who use Strategy 3 or 4 have the same information with respect to the market as investors who use Strategy 2, but they do not use it due to costs of estimation of the factor and they adopt their intuition to the growth rate of the risky security.

(iii) Differences of certainty equivalents between Strategy 3 and 4 can be regarded as difference of investor's investing sense, since in this paper, investors who adopt Strategy 4 use the mean reverting level as the growth rate of the risky security, so that the investors can be considered as investors who have better intuition to the future, on the other hand investors who adopt Strategy 3 use the initial value of the factor as the growth rate of the risky security, so that the investors stand the starting condition of the factor value and do not have a good future vision in our parameter set.

(iv) Differences of certainty equivalents between Strategy 1 and 5 can be regarded as losses from restrictions of investment proportions under full information.

(v) Like (iv), differences of certainty equivalents between Strategy 2 and 6 can be regarded as losses from restrictions of investment proportions under partial information.

- On interest rate $r$ : In Fig.1 and Fig.2, we show relationships between the interest rate $r$ and certainty equivalents which are simulated using Strategy 1-6.

From Fig.1, we can find that the interest rate does not affect to the terminal expected utilities so much. And the order is preserved as Stra. $1>$ Stra. $5>>$ Stra. $=$ Stra.6 $>$ Stra. $4>$ Stra. 3 when $r \in[0,0.1]$.

In Fig.2, the order among strategies in Fig. 1 is broken down, namely when the interest rate is unrealistically high, the order of Stra.5 and 6 is lower than the others due to restrictions of the proportion of the risky security.

- On volatility $\sigma$ : In Fig.3, we show relationships between volatility $\sigma$ and certainty equivalents which are simulated using Strategy 1-6. 


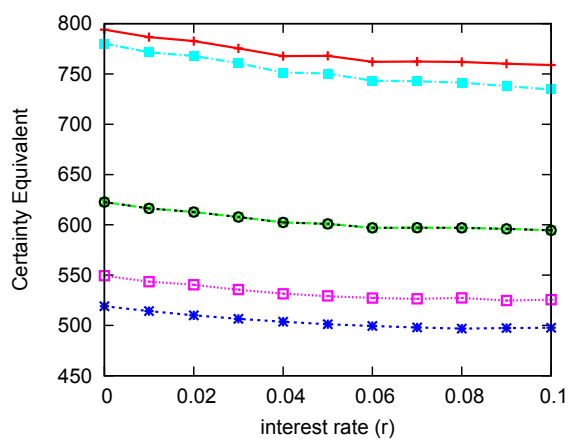

Strategy 1 Strategy $3 \cdots * \cdots$ Strategy 4 Strategy $6 \cdots \cdot \cdot \cdot \cdot$

Fig. 1: Relationships between the interest rate $r$ and certainty equivalents with Strategy 1-6 $(0 \leq r \leq 0.1)$

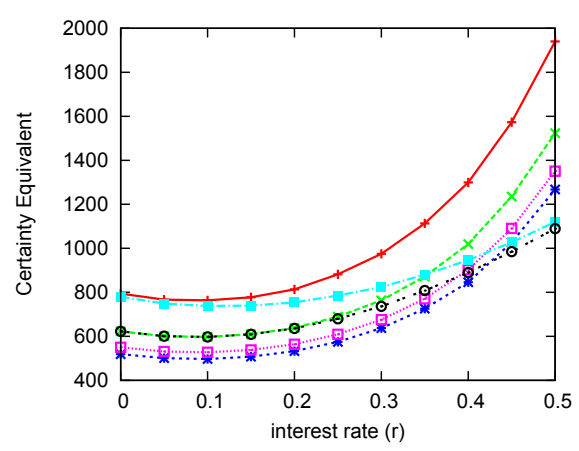

Strategy $1-1$ Strategy $3 \ldots *$...

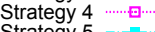
Strategy 5 -......

Fig. 2: Relationships between the interest rate $r$ and certainty equivalents with Strategy 1-6 $(0 \leq r \leq 0.5)$

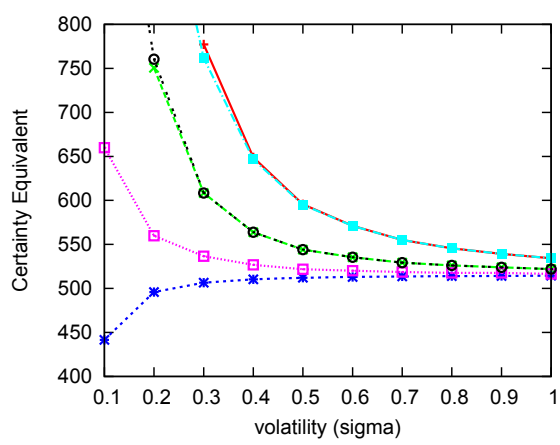

Strategy 1 Strategy 2 ---x-Strategy 3 - …*... Strategy 5 -

Fig. 3: Relationships between volatility $\sigma$ and certainty equivalents with Strategy 1-6
From Fig.3, we can find that when the volatility is really high like the Riemann shock, the differences of certainty equivalents almost disappear.

- On speed of mean reversion $\alpha$ : In Fig.4, we show relationships between speed of mean reversion $\alpha$ and certainty equivalents which are simulated using Strategy 1-6.

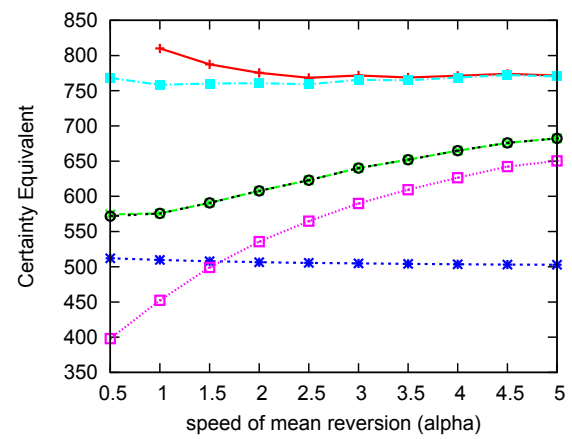

trategy $1-$ Strategy $2-\cdots-\cdots$ Strategy $3 \cdots \cdots \cdots$ Strategy $6 \cdots \cdot \bullet \cdot$

Fig. 4: Relationships between speed of mean reversion $\alpha$ and certainty equivalents with Strategy 1-6

From Fig.4, we can find that when the speed of mean reversion is high, then the differences between full information and partial information are reduced since when it is high, we can guess that the factor strongly fluctuate around the mean reverting level and we can easily obtain highly accurate estimation values through the filtering.

- On mean reverting level $v$ : In Fig.5, we show relationships between mean reverting level $v$ and certainty equivalents which are simulated using Strategy 1-6.

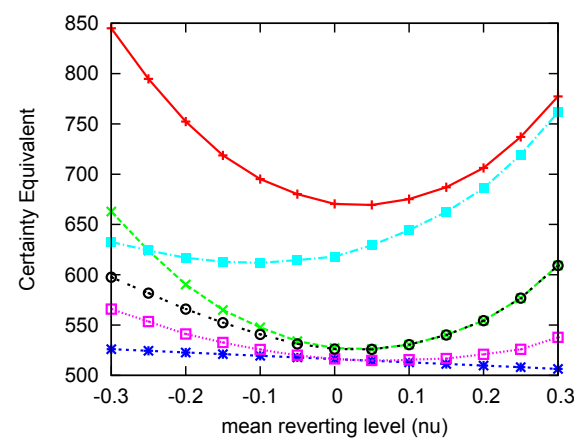

Strategy 1 Strategy $3 \quad \cdots * \cdots$

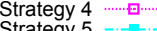
Strategy 5 -

Fig. 5: Relationships between mean reverting level $v$ and certainty equivalents with Strategy 1-6

From Fig.5, we can find that when the mean reverting level is smaller and smaller, differences between Strategy 1 and 5 and between Strategy 2 and 6 are increasing because of the restrictions of investment proportions of the risk security, especially the lower restriction is $l=-1$ now. 
- On volatility of the factor $\beta$ : In Fig.6, we show relationships between volatility of the factor $\beta$ and certainty equivalents which are simulated using Strategy $1-6$.
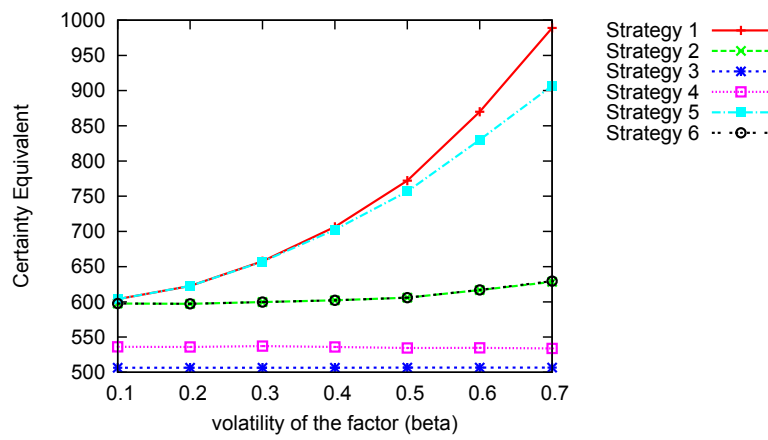

Fig. 6: Relationships between volatility of the factor $\beta$ and certainty equivalents with Strategy 1-6

From Fig.6, we can find that when the volatility of the factor is small, then terminal expected utilities under full and partial information are almost the same. However when it becomes bigger, difference between full and partial information is getting enough bigger since when the volatility of the factor is big, fluctuations of the factor are rough and it is difficult to estimate it through filtering.

- On upper restriction $u$ : In Fig.7 and Fig.8, we show relationships between upper restriction $u$ and certainty equivalents which are simulated using Strategy 1-6, with $l=-1,0$. Note that $l=0$ means that short selling of the risky security are prohibited.
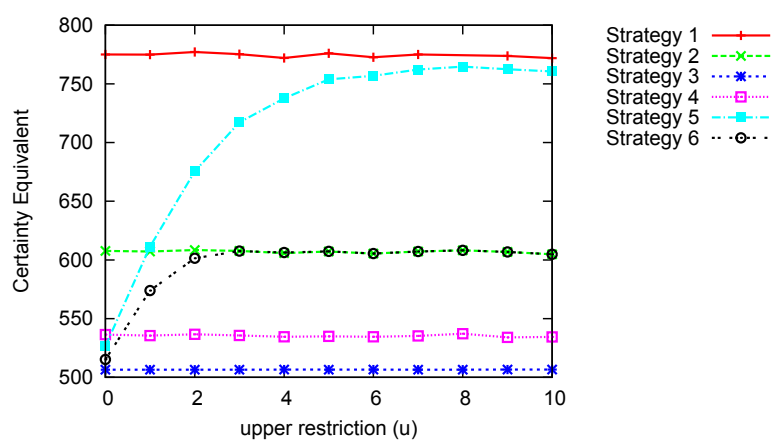

Fig. 7: Relationships between upper restriction $u$ and certainty equivalents with Strategy 1-6 $(l=-1)$

From Fig.7, we can find that Strategy 1 (full information case) is more affected from the upper restriction than Strategy 2 (partial information case), since certainty equivalent of Strategy 5 (full information case with restrictions) is smaller than certainty equivalent of Strategy 1 on $[0,10]$, on the other hand certainty equivalent of Strategy 6 (partial information case with restrictions) is visibly smaller than certainty equivalent of Strategy 2 only on [0,2].

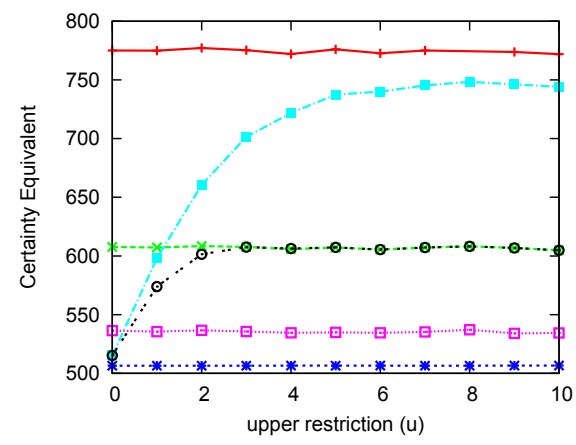

Strategy 1 Strategy $3 \cdots * \cdots$ Strategy 4 Strategy $6 \cdots \cdot \cdot \cdot \cdot$

Fig. 8: Relationships between upper restriction $u$ and certainty equivalents with Strategy 1-6 $(l=0)$

Fig. 8 shows the similar results to Fig.7, that is, certainty equivalent with full information (Strategy 1 ) is more affected by upper restriction comparing to partial information case (Strategy 2) even if lower restriction is replaced by $l=0$ (no short sale).

- On lower restriction $l$ : In Fig.9 and Fig.10, we show a relationship between lower restriction $l$ and certainty equivalents, which are simulated using Strategy 1-6, with $u=6,1$.

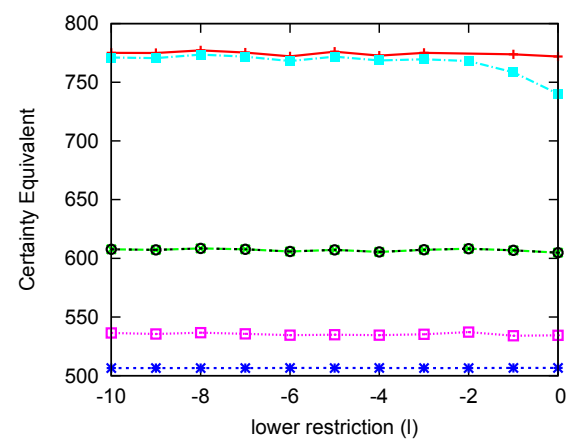

Strategy 1 trategy 3 …*.. Strategy 4 Strategy 6 -

Fig. 9: Relationship between lower restriction $l$ and certainty equivalent with Strategy 1-6 $(u=6)$

From Fig.9, we can find that certainty equivalent with full information (Strategy 1) is more affected by lower restriction than partial information case (Strategy 2) as Fig.7 and Fig.8. And in our set of parameter values, especially $v=0.2$, lower restriction does not reduce certainty equivalent comparing to upper restriction.

From Fig.10, upper restriction $u=1$, namely no short sale of risk-free asset, restricts certainty equivalent much more than the case $u=6$ in Fig.9, so that decrease of certainty equivalent is serious, in particular the full information case (Strategy 1). 


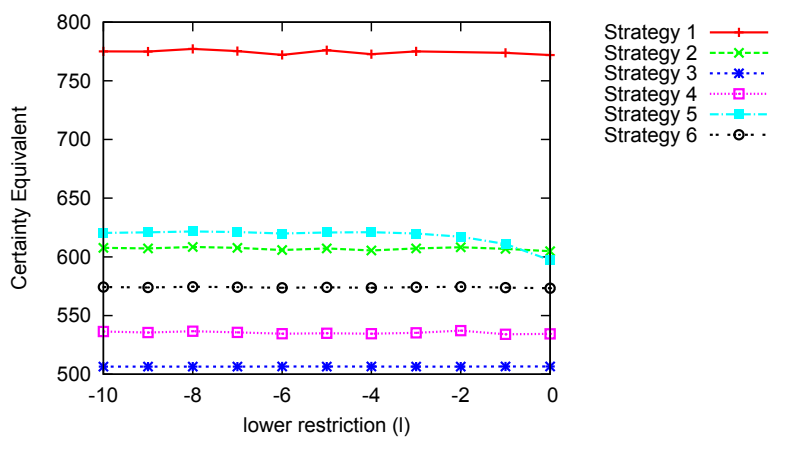

Fig. 10: Relationships between lower restriction $l$ and certainty equivalents with Strategy 1-6 $(u=1)$

\subsection{Histogram of terminal wealth, fundamental statis- tics and standings}

In this section, we give histograms of terminal wealth, fundamental statistics and standings of each two strategies.

Fig. 11 is $10^{5}$ times histograms of terminal expected utilities with each strategy and Fig.12 shows an enlarged view of Fig. 11.
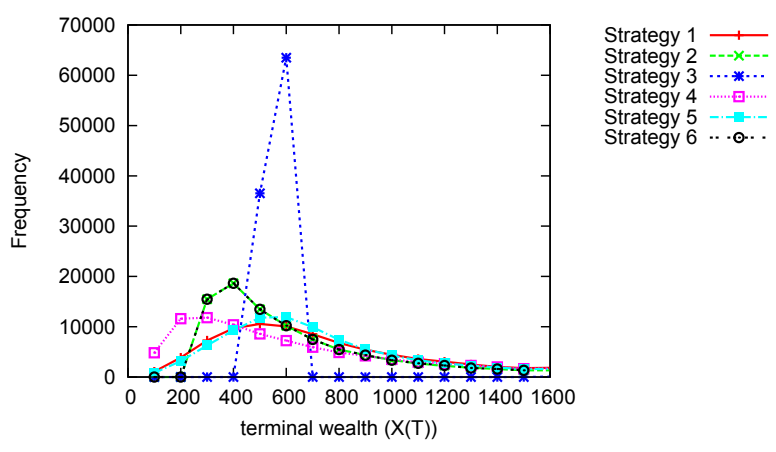

Fig. 11: Histogram with Strategy 1-6

From Fig.11, we can find that distribution of Strategy 3 does not have spread. In Fig.12, distributions of terminal wealth with Strategy 2 and 6 are almost the same and distributions of terminal wealth with Strategy 1 and 5 are close, but somehow different. Comparing distributions of terminal wealth of full and partial information (Strategy 1 and 2), distribution of Strategy 1 has wider spread than distribution of Strategy 2.

In Table 1, we give fundamental statistics of log-return of terminal wealth from time 0 to time $T$ with Strategy 1-6.

From Table 1, the ranking of means (return of portfolio) is Stra. $1>$ Stra. $5>$ Stra. $=$ Stra. $6>$ Stra. $4>$ Stra.3, how ever the ranking of standard deviation (risk of portfolio) is Stra. $4>$ Stra. $1>$ Stra. $5>$ Stra. $=$ Stra.6 $>$ Stra.3. Therefore Strategy 4 has high risk and low return that an investor who uses Strategy 4 is the worst among them. Comparing Strategy 1 and 5, we can find that restrictions of proportion of risky security affects to distribution of terminal wealth's logreturn under full information. However comparing Strategy

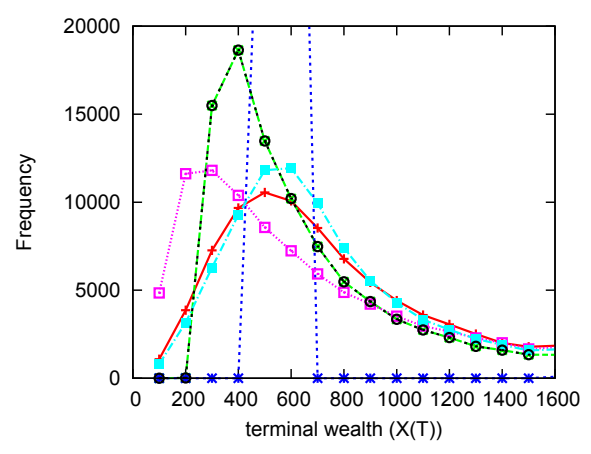

Strategy $1-1-1$
Strategy $2--x--$ Strategy $3 \cdots * \cdots$ Strategy 4 Strategy 6 -

Fig. 12: Histogram with Strategy 1-6 (enlarged view of Fig.11)

2 and 6, they do not affect to distribution of terminal wealth's log-return under partial information.

Table 2 shows ranking of each scenario in $10^{5}$ times Monte-Carlo among Strategy 1-6. Sums of components of each row are not always $10^{5}$ because of draws.

From Table 2, Strategy 1, as expected, wins the most among six strategies, strategies under partial information (Strategy 2 and 6) never become the lowest rank among six strategies surprisingly, and constant strategies (Strategy 3 and 4) are the worst two among them.

Table 3 shows standing of each scenario in $10^{5}$ times Monte-Carlo among Strategy 1-6.

As expected, Strategy 1 has more wins than losses to any other strategies, however in our parameter set the winning rates are about $65 \%$. Notice that between Strategy 1 and 5, sum of wins of each strategy is not equal to $10^{5}$ due to draws. However Strategy 5 wins more than Strategy 1 to Strategy 2-4 and 6. Strategy 2 slightly has more wins than losses to constant proportion strategies (Strategy 3 and 4). As you can see from Table 3, most of matches between Strategy 2 and 6 are draw.

\section{Conclusions}

In this paper, we considered six strategies with respect to expected utility maximization problem under various situations, namely about information, estimation and restrictions. We evaluated these values through certainty equivalents. At first we numerically gave relationships between each parameter and certainty equivalent. From those simulations, we found that values of information were much larger than costs of estimation and losses by restrictions. And we also found that restrictions more affected to full information case than partial information case. Then we considered terminal wealth with each strategy from statistical point of view, namely histograms and fundamental statistics. We found that distributions of terminal wealth under partial information are much different from distribution under full information without restrictions. Finally we analyzed scenario based ranking and standing. Through them we found different aspects of each strategy from results of expected utilities 
Table 1: Fundamental statistics of log-return of terminal wealth with Strategy 1-6

\begin{tabular}{|c|c|c|c|}
\hline & Stra.1 & Stra.2 & Stra.3 \\
\hline Mean & 0.438 & 0.195 & 0.0129 \\
\hline Standard Error & 0.00311 & 0.00234 & 0.000118 \\
\hline Median & 0.317 & 0.0412 & 0.0130 \\
\hline Mood & 0.860 & -0.504 & 0.0270 \\
\hline Standard Deviation & 0.983 & 0.740 & 0.0373 \\
\hline Kurtosis & 3.38 & 2.06 & 0.000152 \\
\hline Skewness & 0.970 & 1.23 & -0.00426 \\
\hline Min. & -5.25 & -0.972 & -0.153 \\
\hline Max. & 10.0 & 5.92 & 0.186 \\
\hline $99 \%$ percentile & 3.57 & 2.52 & 0.0992 \\
\hline \multirow[t]{2}{*}{$1 \%$ percentile } & -1.65 & -0.803 & -0.0737 \\
\hline & Stra.4 & Stra.5 & Stra.6 \\
\hline Mean & 0.0702 & 0.419 & 0.195 \\
\hline Standard Error & 0.00319 & 0.00286 & 0.00234 \\
\hline Median & 0.0691 & 0.285 & 0.0412 \\
\hline Mood & 0.750 & 0.744 & -0.504 \\
\hline Standard Deviation & 1.01 & 0.904 & 0.740 \\
\hline Kurtosis & 0.00136 & 2.59 & 2.03 \\
\hline Skewness & -0.00714 & 0.919 & 1.22 \\
\hline Min. & -4.65 & -4.38 & -0.972 \\
\hline Max. & 4.50 & 7.32 & 5.67 \\
\hline 99\% percentile & 2.40 & 3.28 & 2.52 \\
\hline $1 \%$ percentile & -2.28 & -1.52 & -0.803 \\
\hline
\end{tabular}

Table 2: Ranking of each scenario among Strategy 1-6

\begin{tabular}{c|cccccc}
\hline Rank & Stra.1 & Stra.2 & Stra.3 & Stra.4 & Stra.5 & Stra.6 \\
\hline \hline 1 & 35146 & 7534 & 22615 & 21254 & 16688 & 7642 \\
\hline 2 & 21330 & 19595 & 5535 & 4256 & 40733 & 19532 \\
\hline 3 & 10789 & 19317 & 18105 & 18055 & 12097 & 19591 \\
\hline 4 & 8850 & 43101 & 8035 & 9646 & 12491 & 42893 \\
\hline 5 & 12346 & 10453 & 5877 & 12471 & 15184 & 10342 \\
\hline 6 & 11539 & 0 & 39833 & 34318 & 2807 & 0 \\
\hline
\end{tabular}

(certainty equivalent).

As future works, we need to study effects of parameter estimation errors on expected utility since in partial information case we need to estimate parameters of factors in order to obtain filter values. We need to similarly analyze in the case that Brownian motion of the risky security and Brownian motion of the factor have a correlation. And we try to explicitly calculate several equations and would like to capture features of each strategy from analytical points of view.

\section{References}

[1] Y. Asakura and K. Yasuda: On Effects of Estimations and Information for Expected Log-Utility Maximization Problems, Proceedings of the 45th ISCIE International Symposium on Stochastic Systems Theory and Its Applications (electronic proceedings), pp.9099, 2014.
Table 3: Standing of each scenario among Strategy 1-6

\begin{tabular}{c|cccccc}
\hline win $\backslash$ lost & Stra.1 & Stra.2 & Stra.3 & Stra.4 & Stra.5 & Stra.6 \\
\hline \hline Stra.1 & - & 66815 & 66241 & 65631 & 48112 & 66815 \\
\hline Stra.2 & 33185 & - & 51523 & 55233 & 31487 & 191 \\
\hline Stra.3 & 33759 & 48477 & - & 47818 & 32945 & 48478 \\
\hline Stra.4 & 34369 & 44767 & 52182 & - & 33138 & 44766 \\
\hline Stra.5 & 42039 & 68513 & 67055 & 66862 & - & 68511 \\
\hline Stra.6 & 33185 & 772 & 51522 & 55234 & 31489 & - \\
\hline
\end{tabular}

[2] J. Cvitanić and I. Karatzas: Convex duality in constrained portfolio optimization, Ann. Appl. Probab., Vol.2, pp.767-818, 1992.

[3] I. Karatzas, J.P. Lehoczky, S.E. Shreve and G.-L. Xu: Martingale and duality methods for utility maximization in an incomplete market, SIAM J. Control and Optimization, Vol.29, pp.702-730, 1991.

[4] P. Lakner: Utility Maximization with Partial Information, Stochastic Processes and their Applications, Vol.56, pp.247-249, 1995.

[5] P. Lakner: Optimal trading strategy for an investor: the case of partial information, Stochastic Processes and their Applications, Vol.76, pp.77-97, 1998.

[6] W. Putschögl and J. Sass: Optimal consumption and investment under partial information, Decisions Econ. Finan., Vol.31, pp.137-170, 2008.

[7] J. Sass: Utility Maximization with Convex Constraints and Partial Information, Acta Applicandae Mathematicae, Vol.97, pp.221-238, 2007.

[8] Z. Yang, C.-O. Ewald and W.-K. Wang: A comparative analysis of the value of information in a continuous time market model with partial information: the cases of log-utility and CRRA, Journal of Probability and Statistics, Vol.2011, Article ID 238623, 23pages, 2011. 